\title{
Variational Formulation for the Stationary Fractional Advection Dispersion Equation
}

\author{
Vincent J. Ervin *
}

\author{
John Paul Roop ${ }^{\dagger}$
}

\begin{abstract}
In this paper a theoretical framework for the Galerkin finite element approximation to the steady state fractional advection dispersion equation is presented. Appropriate fractional derivative spaces are defined and shown to be equivalent to the usual fractional dimension Sobolev spaces $H^{s}$. Existence and uniqueness results are proven, and error estimates for the Galerkin approximation derived. Numerical results are included which confirm the theoretical estimates.
\end{abstract}

Key words. Finite element method, fractional differential operator, fractional diffusion equation, fractional advection dispersion equation.

AMS Mathematics subject classifications. 65N30, 35J99

\section{Introduction}

In this paper, we investigate the Galerkin approximation to the steady state Fractional Advection Dispersion Equation (FADE)

$$
-D a\left(p_{0} D_{x}^{-\beta}+q{ }_{x} D_{1}^{-\beta}\right) D u+b(x) D u+c(x) u=f
$$

where $D$ represents a single spatial derivative, and ${ }_{0} D_{x}^{-\beta},{ }_{x} D_{1}^{-\beta}$ represent left and right fractional integral operators, respectively, with $0 \leq \beta<1$, and $0 \leq p, q \leq 1$, satisfying $p+q=1$.

Our interest in (1) arises from its application as a model for physical phenomena exhibiting anomalous diffusion, i.e. diffusion not accurately modeled by the usual advection dispersion equation. Anomalous diffusion has been used in modeling turbulent flow [4, 12], and chaotic dynamics of classical conservative systems [14]. In viscoelasticity, fractional differential operators have been used to describe materials' constitutive equations [7]. Recently articles involving fractional differential operators have appeared in

*Department of Mathematical Sciences, Clemson University, Clemson, SC 29634-0975, email: vjervin@clemson.edu. Partially supported by the National Science Foundation under Award Number DMS-0410792.

${ }^{\dagger}$ Corresponding author. Department of Mathematics, Virginia Tech, Blacksburg, VA 24061-0123. email: jroop@vt.edu. 
Physics Today [13], and Nature [6]. The application of central interest to us is that of contaminant transport in groundwater flow. In [2] the authors state that solutes moving through aquifers do not generally follow a Fickian, second-order, governing equation because of large deviations from the stochastic process of Brownian motion. This give rise to superdiffusive motion.

Fractional differential operators have a long history, having been mentioned by Leibniz in a letter to L'Hospital in 1695. Early mathematicians who contributed to the study of fractional differential operators include Liouville, Riemann and Holmgrem. (See [8] for a history of the development of fractional differential operators). A number of definitions for the fractional derivative has emerged over the years: Grüwald-Letnikov fractional derivative, Riemann-Liouville fractional derivative, and the Caputo fractional derivative [9]. In this article we restrict our attention to the use the Riemann-Liouville fractional derivative.

To date most solution techniques for equations involving fractional differential operators have exploited the properties of the Fourier and Laplace transforms of the operators to determine a classical solution. Finite difference have also been applied to construct numerical approximation [9]. Aside from [5] we are not aware of any other papers in the literature which investigate the Galerkin approximation and associated error analysis for the FADE.

There are two properties of fractional differential operators which make the analysis of the variational solution to the FADE more complicated than than that for the usual advection dispersion equation. These are

(i) fractional differential operators are not local operators, and

(ii) the adjoint of a fractional differential operator is not the negative of itself.

Because of (i) and (ii) the correct function space setting for the variational solution is not obvious. Related

to the left fractional derivative we introduce the $J_{L}^{\mu}$ space and corresponding to the right fractional derivative the $J_{R}^{\mu}$ space. We are able to relate these spaces to the fractional Sobolev space $H^{\mu}$ through an intermediate space $J_{S}^{\mu}$.

This paper is organized as follows. In Section 2 we develop the appropriate functional setting for the variational solution of FADEs. In Section 3 we then prove existence and uniqueness of the variational solution. The Galerkin approximation is introduced in Section 4, and convergence results for the Galerkin approximation are derived. Numerical results demonstrating the convergence of the Galerkin approximation are presented in Section 5. Contained in the Appendix are the definitions of the RiemannLiouville fractional derivative and integral operators, together with some other useful properties of these operators.

\section{Fractional Derivative Spaces}

In this section we develop the abstract setting for the analysis of the approximation to FADEs. We introduce associated left, right, and symmetric fractional derivative spaces. The equivalence of the 
fractional derivative spaces with fractional order Hilbert spaces is then established.

In order to define the spaces, for $G \subset \mathbb{R}$ an open interval (which may be unbounded) we let $C_{0}^{\infty}(G)$ denote the set of all functions $u \in C^{\infty}(G)$ that vanish outside a compact subset $K$ of $G$.

Definition 2.1 [Left Fractional Derivative Space] Let $\mu>0$. Define the semi-norm

$$
|u|_{J_{L}^{\mu}(\mathbb{R})}:=\left\|\mathbf{D}^{\mu} u\right\|_{L^{2}(\mathbb{R})},
$$

and norm

$$
\|u\|_{J_{L}^{\mu}(\mathbb{R})}:=\left(\|u\|_{L^{2}(\mathbb{R})}^{2}+|u|_{J_{L}^{\mu}(\mathbb{R})}^{2}\right)^{1 / 2}
$$

and let $J_{L}^{\mu}(\mathbb{R})$ denote the closure of $C_{0}^{\infty}(\mathbb{R})$ with respect to $\|\cdot\|_{J_{L}^{\mu}(\mathbb{R})}$.

In the following analysis, we define a semi-norm for functions in $H^{\mu}(\mathbb{R})$ in terms of the Fourier transform.

Definition 2.2 Let $\mu>0$. Define the semi-norm

$$
|u|_{H^{\mu}(\mathbb{R})}:=\left\||\omega|^{\mu} \hat{u}\right\|_{L^{2}(\mathbb{R})}
$$

and norm

$$
\|u\|_{H^{\mu}(\mathbb{R})}:=\left(\|u\|_{L^{2}(\mathbb{R})}^{2}+|u|_{H^{\mu}(\mathbb{R})}^{2}\right)^{1 / 2}
$$

and let $H^{\mu}(\mathbb{R})$ denote the closure of $C_{0}^{\infty}(\mathbb{R})$ with respect to $\|\cdot\|_{H^{\mu}(\mathbb{R})}$.

Theorem 2.1 The spaces $J_{L}^{\mu}(\mathbb{R})$ and $H^{\mu}(\mathbb{R})$ are equal with equivalent semi-norms and norms.

Proof. The proof follows immediately from the following lemma.

Lemma 2.2 Let $\mu>0$, be given. A function $u \in L^{2}(\mathbb{R})$ belongs to $J_{L}^{\mu}(\mathbb{R})$ if and only if

$$
|\omega|^{\mu} \hat{u} \in L^{2}(\mathbb{R})
$$

Specifically,

$$
|u|_{J_{L}^{\mu}(\mathbb{R})}=\left\||\omega|^{\mu} \hat{u}\right\|_{L^{2}(\mathbb{R})} .
$$

Proof. Let $u \in J_{L}^{\mu}(\mathbb{R})$ be given. Then $\mathbf{D}^{\mu} u \in L^{2}(\mathbb{R})$, and from (49) and (45)

$$
\mathcal{F}\left(\mathbf{D}^{\mu} u\right)=(i \omega)^{\mu} \hat{u}
$$

Using Plancherel's theorem, we have

$$
\int_{\mathbb{R}}|\omega|^{2 \mu}|\hat{u}|^{2} d \omega=\int_{\mathbb{R}}\left|\mathbf{D}^{\mu} u\right|^{2} d x .
$$

Hence,

$$
\left\||\omega|^{\mu} \hat{u}\right\|_{L^{2}(\mathbb{R})}=|u|_{J_{L}^{\mu}(\mathbb{R})}
$$

Analogous to $J_{L}^{\mu}(\mathbb{R})$ we introduce $J_{R}^{\mu}(\mathbb{R})$, the right fractional derivative space, and establish their equivalence. 
Definition 2.3 [Right Fractional Derivative Space] Let $\mu>0$. Define the semi-norm

$$
|u|_{J_{R}^{\mu}(\mathbb{R})}:=\left\|\mathbf{D}^{\mu *} u\right\|_{L^{2}(\mathbb{R})},
$$

and norm

$$
\|u\|_{J_{R}^{\mu}(\mathbb{R})}:=\left(\|u\|_{L^{2}(\mathbb{R})}^{2}+|u|_{J_{R}^{\mu}(\mathbb{R})}^{2}\right)^{1 / 2}
$$

and let $J_{R}^{\mu}(\mathbb{R})$ denote the closure of $C_{0}^{\infty}(\mathbb{R})$ with respect to $\|\cdot\|_{J_{R}^{\mu}(\mathbb{R})}$.

Theorem 2.3 Let $\mu>0$. The spaces $J_{L}^{\mu}(\mathbb{R})$ and $J_{R}^{\mu}(\mathbb{R})$ are equal, with equivalent semi-norms and norms.

Proof. We need only verify that the $J_{L}(\mathbb{R})$ and $J_{R}(\mathbb{R})$ semi-norms are equivalent. This is done using the Fourier transform. Combining (44), the definitions of $\mathbf{D}^{\mu}, \mathbf{D}^{\mu *}$, and Plancherel's theorem yields

$$
\begin{aligned}
|u|_{J_{L}^{\mu}(\mathbb{R})}^{2} & =\int_{\mathbb{R}}\left|(i \omega)^{\mu} \hat{u}(\omega)\right|^{2} d \omega \\
|u|_{J_{R}^{\mu}(\mathbb{R})}^{2} & =\int_{\mathbb{R}}\left|(-i \omega)^{\mu} \hat{u}(\omega)\right|^{2} d \omega .
\end{aligned}
$$

Thus the semi-norms are equivalent, and, in fact, equal as $\left|(i \omega)^{\mu}\right|=\left|(-i \omega)^{\mu}\right|$.

In the finite element analysis of (1), we make use of the bilinear functional $\left(\mathbf{D}^{\mu} \cdot, \mathbf{D}^{\mu *} \cdot\right)$. For the case of the entire real line, we can relate this mapping to $|\cdot|_{J_{L}^{\mu}(\mathbb{R})}$.

Lemma 2.4 Let $\mu>0, n$ be the smallest integer greater than $\mu(n-1 \leq \mu<n)$, and $\sigma=n-\mu$. Then for $u(x)$ a real valued function

$$
\left(\mathbf{D}^{\mu} u, \mathbf{D}^{\mu *} u\right)=\cos (\pi \mu)\left\|\mathbf{D}^{\mu} u\right\|_{L^{2}(\mathbb{R})}^{2} .
$$

Proof. Helpful in establishing this result is the Fourier transform property (- denotes complex conjugate)

$$
\int_{\mathbb{R}} u \bar{v} d x=\int_{\mathbb{R}} \hat{u} \bar{v} d \omega
$$

and the observation that

$$
\overline{(i \omega)^{\mu}}=\left\{\begin{array}{cc}
\exp (-i \pi \mu) \overline{(-i \omega)^{\mu}} & \text { if } \omega \geq 0 \\
\exp (i \pi \mu) \overline{(-i \omega)^{\mu}} & \text { if } \omega<0
\end{array} .\right.
$$

Thus

$$
\begin{aligned}
\left(\mathbf{D}^{\mu} u, \mathbf{D}^{\mu *} u\right)= & \left(D^{n}{ }_{-\infty} D_{x}^{-\sigma} u, \overline{(-D)^{n}{ }_{x} D_{\infty}^{-\sigma} u}\right) \\
= & \left((i \omega)^{\mu} \hat{u}, \overline{(-i \omega)^{\mu} \hat{u}}\right) \\
= & \int_{-\infty}^{0}(i \omega)^{\mu} \hat{u} \overline{(-i \omega)^{\mu} \hat{u}} d \omega \\
& +\int_{0}^{\infty}(i \omega)^{\mu} \hat{u} \overline{(-i \omega)^{\mu} \hat{u}} d \omega .
\end{aligned}
$$

Using (11) this becomes

$$
\left(\mathbf{D}^{\mu} u, \mathbf{D}^{\mu *} u\right)=\int_{-\infty}^{0}(i \omega)^{\mu} \hat{u} \exp (-i \pi \mu) \overline{(i \omega)^{\mu} \hat{u}} d \omega
$$




$$
\begin{gathered}
+\int_{0}^{\infty}(i \omega)^{\mu} \hat{u} \exp (i \pi \mu) \overline{(i \omega)^{\mu} \hat{u}} d \omega \\
=\cos (\pi \mu) \int_{-\infty}^{\infty}(i \omega)^{\mu} \hat{u} \overline{(i \omega)^{\mu} \hat{u}} d \omega \\
+i \sin (\pi \mu)\left(\int_{0}^{\infty}(i \omega)^{\mu} \hat{u} \overline{(i \omega)^{\mu} \hat{u}} d \omega\right. \\
\left.\quad-\int_{-\infty}^{0}(i \omega)^{\mu} \hat{u} \overline{(i \omega)^{\mu} \hat{u}} d \omega\right) .
\end{gathered}
$$

For $f(x)$ real we have that $\overline{\mathcal{F}(f)(-\omega)}=\mathcal{F}(f)(\omega)$. Thus

$$
\int_{-\infty}^{0}(i \omega)^{\mu} \hat{u} \overline{(i \omega)^{\mu} \hat{u}} d \omega=\int_{0}^{\infty}(i \omega)^{\mu} \hat{u} \overline{(i \omega)^{\mu} \hat{u}} d \omega
$$

Therefore, combining (12) and (13) we obtain

$$
\left(\mathbf{D}^{\mu} u, \mathbf{D}^{\mu *} u\right)=\cos (\pi \mu)\left(\mathbf{D}^{\mu} u, \mathbf{D}^{\mu} u\right) .
$$

Remark. Note that for $\mu=n-1 / 2, n \in \mathbb{N},\left(\mathbf{D}^{\mu} u, \mathbf{D}^{\mu *} u\right)=0$. For example, $\left(\mathbf{D}^{1 / 2} u, \mathbf{D}^{1 / 2 *} u\right)=$ $(D u, u)=0$, provided that $u$ vanishes on the boundary.

Definition 2.4 [Symmetric Fractional Derivative Space] Let $\mu>0, \mu \neq n-1 / 2, n \in \mathbb{N}$. Define the semi-norm

$$
|u|_{J_{S}^{\mu}(\mathbb{R})}:=\left|\left(\mathbf{D}^{\mu} u, \mathbf{D}^{\mu *} u\right)_{L^{2}(\mathbb{R})}\right|^{1 / 2},
$$

and norm

$$
\|u\|_{J_{S}^{\mu}(\mathbb{R})}:=\left(\|u\|_{L^{2}(\mathbb{R})}^{2}+|u|_{J_{S}^{\mu}(\mathbb{R})}^{2}\right)^{1 / 2}
$$

and let $J_{S}^{\mu}(\mathbb{R})$ denote the closure of $C_{0}^{\infty}(\mathbb{R})$ with respect to $\|\cdot\|_{J_{S}^{\mu}(\mathbb{R})}$.

Theorem 2.5 For $\mu>0, \mu \neq n-1 / 2, n \in \mathbb{N}$, the spaces $J_{L}^{\mu}(\mathbb{R})$ and $J_{S}^{\mu}(\mathbb{R})$ are equal, with equivalent semi-norms and norms.

Proof. We must show that the $J_{L}^{\mu}(\mathbb{R})$ and $J_{S}^{\mu}(\mathbb{R})$ semi-norms are equivalent. We have from the previous lemma that

$$
|u|_{J_{S}^{\mu}(\mathbb{R})}^{2}=|\cos (\pi \mu)||u|_{J_{L}^{\mu}(\mathbb{R})}^{2}
$$

Let $\Omega=(l, r)$ be a bounded open subinterval of $\mathbb{R}$. We now restrict the fractional derivative spaces to $\Omega$.

Definition 2.5. Define the spaces $J_{L, 0}^{\mu}(\Omega), J_{R, 0}^{\mu}(\Omega), J_{S, 0}^{\mu}(\Omega)$ as the closures of $C_{0}^{\infty}(\Omega)$ under their respective norms.

Following are several useful intermediate results which we use in order to relate the spaces $J_{L, 0}^{\mu}(\Omega), J_{R, 0}^{\mu}(\Omega)$, and $J_{S, 0}^{\mu}(\Omega)$ to the fractional Sobolev space $H_{0}^{\mu}(\Omega)$.

Lemma 2.6 Let $\mu>0$. The following mapping properties hold.

(i) $\mathbf{D}^{-\mu}: L^{2}(\Omega) \rightarrow L^{2}(\Omega)$ is a bounded linear operator. 
(ii) $\mathbf{D}^{-\mu}: L^{2}(\Omega) \rightarrow J_{L}^{\mu}(\Omega)$ is a bounded linear operator.

(iii) $\mathbf{D}^{\mu}: J_{L}^{\mu}(\Omega) \rightarrow L^{2}(\Omega)$ is a bounded linear operator.

(iv) $\mathbf{D}^{-\mu *}: L^{2}(\Omega) \rightarrow L^{2}(\Omega)$ is a bounded linear operator.

$(\mathrm{v}) \mathbf{D}^{-\mu *}: L^{2}(\Omega) \rightarrow J_{R}^{\mu}(\Omega)$ is a bounded linear operator.

(vi) $\mathbf{D}^{\mu *}: J_{R}^{\mu}(\Omega) \rightarrow L^{2}(\Omega)$ is a bounded linear operator.

Proof. In order to prove (i) we note that $\mathbf{D}^{-\mu} u=\frac{{ }^{\mu} \mu-1}{\Gamma(\mu)} * u$, where $*$ denotes convolution. Using Young's theorem [1],

$$
\|v * w\|_{L^{p}(\Omega)} \leq\|v\|_{L^{1}(\Omega)}\|w\|_{L^{p}(\Omega)}, 1 \leq p<\infty
$$

we have

$$
\begin{aligned}
\left\|\mathbf{D}^{-\mu} u\right\|_{L^{2}(\Omega)} & =\frac{1}{\Gamma(\mu)}\left\|x^{\mu-1} * u\right\|_{L^{2}(\Omega)} \\
& \leq \frac{1}{\Gamma(\mu)}\left\|x^{\mu-1}\right\|_{L^{1}(\Omega)}\|u\|_{L^{2}(\Omega)} \\
& =\frac{1}{\Gamma(\mu)} \int_{\Omega}|x|^{\mu-1} d x\|u\|_{L^{2}(\Omega)} \\
& \leq \frac{|r|^{\mu}+|l|^{\mu}}{\Gamma(\mu+1)}\|u\|_{L^{2}(\Omega)} .
\end{aligned}
$$

From the definition of $J_{L}^{\mu}(\Omega)$ and using Property A.4 (in the Appendix), we have

$$
\begin{aligned}
\left\|\mathbf{D}^{-\mu} u\right\|_{J_{L}^{\mu}(\Omega)} & =\left(\left\|\mathbf{D}^{-\mu} u\right\|_{L^{2}(\Omega)}^{2}+\left\|\mathbf{D}^{\mu} \mathbf{D}^{-\mu} u\right\|_{L^{2}(\Omega)}^{2}\right)^{1 / 2} \\
& =\left(\left\|\mathbf{D}^{-\mu} u\right\|_{L^{2}(\Omega)}^{2}+\|u\|_{L^{2}(\Omega)}^{2}\right)^{1 / 2}
\end{aligned}
$$

Then, using (i), property (ii) follows.

The result (iii) follows directly from the definition of the $J_{L}^{\mu}(\Omega)$ norm as

$$
\left\|\mathbf{D}^{\mu} u\right\|_{L^{2}(\Omega)} \leq\left(\|u\|_{L^{2}(\Omega)}^{2}+\left\|\mathbf{D}^{\mu} u\right\|_{L^{2}(\Omega)}^{2}\right)^{1 / 2} .
$$

Finally, the proofs of (iv) - (vi) are analogous to the proofs of (i) - (iii).

Lemma 2.7 For $u \in J_{L, 0}^{\mu}(\Omega)$, we have $\mathbf{D}^{-\mu} \mathbf{D}^{\mu} u=u$, and for $u \in J_{R, 0}^{\mu}(\Omega)$, we have $\mathbf{D}^{-\mu *} \mathbf{D}^{\mu *} u=u$.

Proof. By the definition of $J_{L, 0}^{\mu}(\Omega)$, there exists a sequence $\left\{\phi_{n}\right\}_{n=1}^{\infty} \subset C_{0}^{\infty}(\Omega)$ such that

$$
\lim _{n \rightarrow \infty}\left\|u-\phi_{n}\right\|_{J_{L}^{\mu}(\Omega)}=0
$$

Applying the triangle inequality, we have

$$
\left\|\mathbf{D}^{-\mu} \mathbf{D}^{\mu} u-u\right\|_{J_{L}^{\mu}(\Omega)} \leq\left\|\mathbf{D}^{-\mu} \mathbf{D}^{\mu}\left(u-\phi_{n}\right)\right\|_{J_{L}^{\mu}(\Omega)}+\left\|\mathbf{D}^{-\mu} \mathbf{D}^{\mu} \phi_{n}-\phi_{n}\right\|_{J_{L}^{\mu}(\Omega)}+\left\|\phi_{n}-u\right\|_{J_{L}^{\mu}(\Omega)} .
$$

Since $\phi_{n} \in C_{0}^{\infty}(\Omega)$, Property A.6 (in the Appendix) implies that $\left\|\mathbf{D}^{-\mu} \mathbf{D}^{\mu} \phi_{n}-\phi_{n}\right\|_{J_{L}^{\mu}(\Omega)}=0$. By the mapping properties in Lemma 2.6,

$$
\left\|\mathbf{D}^{-\mu} \mathbf{D}^{\mu}\left(u-\phi_{n}\right)\right\|_{J_{L}^{\mu}(\Omega)} \leq C\left\|u-\phi_{n}\right\|_{J_{L}^{\mu}(\Omega)}
$$

Thus,

$$
\left\|\mathbf{D}^{-\mu} \mathbf{D}^{\mu} u-u\right\|_{J_{L}^{\mu}(\Omega)} \leq(C+1)\left\|u-\phi_{n}\right\|_{J_{L}^{\mu}(\Omega)}
$$


Taking the limit as $n \rightarrow \infty$, we obtain the stated result for $J_{L, 0}^{\mu}(\Omega)$. The result for $J_{R, 0}^{\mu}(\Omega)$ follows similarly.

Corollary 2.8 For $u \in J_{L, 0}^{\mu}(\Omega), 0<s<\mu$,

$$
\mathbf{D}^{-s} \mathbf{D}^{s} \mathbf{D}^{\mu-s} u=\mathbf{D}^{\mu-s} u
$$

Proof. Proceeding as in the proof of Lemma 2.7,

$$
\begin{aligned}
\left\|\mathbf{D}^{-s} \mathbf{D}^{s} \mathbf{D}^{\mu-s} u-\mathbf{D}^{\mu-s} u\right\|_{L^{2}(\Omega)} \leq & \left\|\mathbf{D}^{-s} \mathbf{D}^{s} \mathbf{D}^{\mu-s}\left(u-\phi_{n}\right)\right\|_{L^{2}(\Omega)} \\
& +\left\|\mathbf{D}^{-s} \mathbf{D}^{s} \mathbf{D}^{\mu-s} \phi_{n}-\mathbf{D}^{\mu-s} \phi_{n}\right\|_{L^{2}(\Omega)} \\
& +\left\|\mathbf{D}^{\mu-s}\left(u-\phi_{n}\right)\right\|_{L^{2}(\Omega)} \\
\leq & \left\|\mathbf{D}^{-s} \mathbf{D}^{\mu}\left(u-\phi_{n}\right)\right\|_{L^{2}(\Omega)}+\left\|u-\phi_{n}\right\|_{J_{L, 0}^{\mu-s}(\Omega)} \\
& +\left\|\mathbf{D}^{-s} \mathbf{D}^{s} \mathbf{D}^{\mu-s} \phi_{n}-\mathbf{D}^{\mu-s} \phi_{n}\right\|_{L^{2}(\Omega)} .
\end{aligned}
$$

An elementary calculation shows that for $\phi_{n} \in C_{0}^{\infty}(\Omega)$,

$$
\mathbf{D}^{\mu-s} \phi_{n}(l)=0
$$

Hence, by Property A.6,

$$
\left\|\mathbf{D}^{-s} \mathbf{D}^{s} \mathbf{D}^{\mu-s} \phi_{n}-\mathbf{D}^{\mu-s} \phi_{n}\right\|_{L^{2}(\Omega)}=0
$$

The stated result then follows from the convergence of $\phi_{n}$ to $u$ and the mapping properties in Lemma 2.6 .

Analogous to fractional integral operators, fractional differential operators also satisfy a semi-group property.

Lemma 2.9 For $u \in J_{L, 0}^{\mu}(\Omega), 0<s<\mu$, we have

$$
\mathbf{D}^{\mu} u=\mathbf{D}^{s} \mathbf{D}^{\mu-s} u
$$

and, similarly for $u \in J_{R, 0}^{\mu}(\Omega)$,

$$
\mathbf{D}^{\mu *} u=\mathbf{D}^{s *} \mathbf{D}^{(\mu-s) *} u
$$

Proof. Let $u \in J_{L, 0}^{\mu}(\Omega)$. Then by Lemma 2.7 and Property A.1, we have

$$
u=\mathbf{D}^{-\mu} \mathbf{D}^{\mu} u=\mathbf{D}^{s-\mu} \mathbf{D}^{-s} \mathbf{D}^{\mu} u
$$

Applying $\mathbf{D}^{s} \mathbf{D}^{\mu-s}$ to both sides and using Property A.4,

$$
\mathbf{D}^{s} \mathbf{D}^{\mu-s} u=\mathbf{D}^{s} \mathbf{D}^{\mu-s} \mathbf{D}^{s-\mu} \mathbf{D}^{-s} \mathbf{D}^{\mu} u=\mathbf{D}^{\mu} u
$$

The result for $J_{R, 0}^{\mu}(\Omega)$ follows analogously.

Theorem 2.10 [Fractional Poincaré-Friedrichs] For $u \in J_{L, 0}^{\mu}(\Omega)$, we have

$$
\|u\|_{L^{2}(\Omega)} \leq C|u|_{J_{L}^{\mu}(\Omega)},
$$


and for $u \in J_{R, 0}^{\mu}(\Omega)$,

$$
\|u\|_{L^{2}(\Omega)} \leq C|u|_{J_{R}^{\mu}(\Omega)} .
$$

Proof. Combining Lemmas 2.6 and 2.7, for $u \in J_{L, 0}^{\mu}(\Omega)$

$$
\|u\|_{L^{2}(\Omega)}=\left\|\mathbf{D}^{-\mu} \mathbf{D}^{\mu} u\right\|_{L^{2}(\Omega)} \leq C\left\|\mathbf{D}^{\mu} u\right\|_{L^{2}(\Omega)}=C|u|_{J_{L}^{\mu}(\Omega)} .
$$

Inequality (17) follows similarly.

Corollary 2.11 For $u \in J_{L, 0}^{\mu}(\Omega), 0<s<\mu$, we have

$$
|u|_{J_{L}^{s}(\Omega)} \leq C|u|_{J_{L}^{\mu}(\Omega)},
$$

and for $u \in J_{R, 0}^{\mu}(\Omega), 0<s<\mu$,

$$
|u|_{J_{R}^{s}(\Omega)} \leq C|u|_{J_{R}^{\mu}(\Omega)} .
$$

Proof. Using Lemma 2.9, for $u \in J_{L, 0}^{\mu}(\Omega)$

$$
\begin{aligned}
\left\|\mathbf{D}^{s} u\right\|_{L^{2}(\Omega)} & =\left\|\mathbf{D}^{s} \mathbf{D}^{-\mu} \mathbf{D}^{\mu} u\right\|_{L^{2}(\Omega)} \\
& =\left\|\mathbf{D}^{s} \mathbf{D}^{-s} \mathbf{D}^{s-\mu} \mathbf{D}^{\mu} u\right\|_{L^{2}(\Omega)} \\
& =\left\|\mathbf{D}^{s-\mu} \mathbf{D}^{\mu} u\right\|_{L^{2}(\Omega)} \\
& \leq C\left\|\mathbf{D}^{\mu} u\right\|_{L^{2}(\Omega)} .
\end{aligned}
$$

The result for $J_{R, 0}^{\mu}(\Omega)$ follows analogously.

We next turn to the equivalence of the fractional derivative spaces $J_{L, 0}^{\mu}(\Omega), J_{R, 0}^{\mu}(\Omega)$, and the fractional order Sobolev spaces $H_{0}^{\mu}(\Omega)$. The analysis of the equivalence of these spaces is complicated by the nonlocalness of the fractional differential operators.

Theorem 2.12 Let $\mu>0, \mu \neq n-1 / 2, n \in \mathbb{N}$. Then the $J_{S, 0}^{\mu}(\Omega)$ and $H_{0}^{\mu}(\Omega)$ spaces are equal, with equivalent semi-norms and norms.

Proof. Let $u \in C_{0}^{\infty}(\Omega)$ and $\tilde{u}$ be the extension of $u$ by zero outside of $\Omega$. Then $\operatorname{supp}(\tilde{u}) \subset(l, r)$. However, as the left and right fractional derivatives are non-local,

$$
\operatorname{supp}\left(\mathbf{D}^{\mu} \tilde{u}\right) \subset(l, \infty)
$$

and

$$
\operatorname{supp}\left(\mathbf{D}^{\mu *} \tilde{u}\right) \subset(-\infty, r) .
$$

Nonetheless, the product $\mathbf{D}^{\mu} u \mathbf{D}^{\mu *} u$ has support in $\Omega=(l, r)$. Hence,

$$
|u|_{J_{S, 0}^{\mu}(\Omega)}=|\tilde{u}|_{J_{S}^{\mu}(\mathbb{R})}
$$

and

$$
|u|_{H_{0}^{\mu}(\Omega)}=|\tilde{u}|_{H^{\mu}(\mathbb{R})} .
$$

From Theorem 2.1 and Theorem 2.5 we have that $J_{S}^{\mu}(\mathbb{R})$ and $H^{\mu}(\mathbb{R})$ are equal with equivalent norms. Thus the norms $\|\cdot\|_{J_{S, 0}^{\mu}(\mathbb{R})}$ and $\|\cdot\|_{H_{0}^{\mu}(\mathbb{R})}$ are also equivalent. Finally, as $J_{S, 0}^{\mu}(\Omega)$ and $H_{0}^{\mu}(\Omega)$ are the closures of $C_{0}^{\infty}(\Omega)$ with respect to equivalent norms, the spaces must also be equal. 
The preceding theorem followed from the fact that, for functions with support restricted to the interior of $\Omega,|u|_{J_{S, 0}^{\mu}(\Omega)}=|\tilde{u}|_{J_{S}^{\mu}(\mathbb{R})}$. This is not the case for the spaces $J_{L, 0}^{\mu}(\Omega)$ where we only have $|u|_{J_{L, 0}^{\mu}(\Omega)} \leq|\tilde{u}|_{J_{L}^{\mu}(\mathbb{R})}$. However, following Theorem 2.13 we show that $|\tilde{u}|_{J_{L}^{\mu}(\mathbb{R})} \leq C|\tilde{u}|_{J_{L}^{\mu}(\Omega)}$, which implies that the contribution to $|\tilde{u}|_{J_{L}^{\mu}(\mathbb{R})}$ from outside of $\Omega$ is bounded by a constant times that from the interior of $\Omega$.

Theorem 2.13 Let $\mu>0$. Then the $J_{L, 0}^{\mu}(\Omega), J_{R, 0}^{\mu}(\Omega)$, and $H_{0}^{\mu}(\Omega)$ spaces are equal. Also, if $\mu \neq$ $n-1 / 2, n \in \mathbb{N}$, the spaces $J_{L, 0}^{\mu}(\Omega), J_{R, 0}^{\mu}(\Omega)$, and $H_{0}^{\mu}(\Omega)$ have equivalent semi-norms and norms.

Proof. Again, let $u \in C_{0}^{\infty}(\Omega)$ and $\tilde{u}$ be the extension of $u$ by zero outside of $\Omega$. From (3) and (8) we have

$$
\left\|\mathbf{D}^{\mu} u\right\|_{L^{2}(\Omega)}=|u|_{J_{L}^{\mu}(\Omega)} \leq|\tilde{u}|_{J_{L}^{\mu}(\mathbb{R})}=|\tilde{u}|_{H^{\mu}(\mathbb{R})}=|u|_{H^{\mu}(\Omega)},
$$

and thus $H_{0}^{\mu}(\Omega) \subseteq J_{L, 0}^{\mu}(\Omega)$.

In order to show the reverse inequality, we use the equivalence of the $J_{S, 0}^{\mu}(\Omega)$ and $H_{0}^{\mu}(\Omega)$ norms for $\mu \neq n-1 / 2, n \in \mathbb{N}$ (Theorem 2.12). Using Young's inequality, we obtain

$$
\begin{aligned}
|u|_{H_{0}^{\mu}(\Omega)}^{2} & \leq C|u|_{J_{S, 0}^{\mu}(\Omega)}^{2} \\
& =C\left|\int_{\Omega} \mathbf{D}^{\mu} u \mathbf{D}^{\mu *} u d x\right| \\
& \leq \frac{C}{4 \epsilon}\left\|\mathbf{D}^{\mu} u\right\|_{L^{2}(\Omega)}^{2}+C \epsilon\left\|\mathbf{D}^{\mu *} u\right\|_{L^{2}(\Omega)}^{2} \\
& =\frac{C}{4 \epsilon}|u|_{J_{L, 0}^{\mu}(\Omega)}^{2}+C \epsilon|u|_{J_{R, 0}^{\mu}(\Omega)}^{2} \\
& \leq \frac{C}{4 \epsilon}|u|_{J_{L, 0}^{\mu}(\Omega)}^{2}+C \epsilon|\tilde{u}|_{J_{R}^{\mu}(\mathbb{R})}^{2} \\
& =\frac{C}{4 \epsilon}|u|_{J_{L, 0}^{\mu}(\Omega)}^{2}+C \epsilon|\tilde{u}|_{H^{\mu}(\mathbb{R})}^{2} \\
& =\frac{C}{4 \epsilon}|u|_{J_{L, 0}^{\mu}(\Omega)}^{2}+C \epsilon|u|_{H_{0}^{\mu}(\Omega)}^{2} .
\end{aligned}
$$

Therefore, taking $\epsilon=1 /(2 C)$, we have

$$
|u|_{H_{0}^{\mu}(\Omega)}^{2} \leq C^{2}|u|_{J_{L, 0}^{\mu}(\Omega)}^{2},
$$

from which we obtain $J_{L, 0}^{\mu}(\Omega) \subseteq H_{0}^{\mu}(\Omega)$.

The equivalence of norms follows then from the equivalence of semi-norms and the definition of the $J_{L, 0}^{\mu}(\Omega)$ and $H_{0}^{\mu}(\Omega)$ norms. The result for $J_{R, 0}^{\mu}(\Omega)$ follows analogously.

Corollary 2.14 Let $\mu \neq n-1 / 2, n \in \mathbb{N}$. Then for $u \in J_{L, 0}^{\mu}(\Omega)$ with $\tilde{u}$ the extension of $u$ by zero outside of $\Omega$ there exists a constant $C$ (independent of $u$ ) such that

$$
|\tilde{u}|_{J_{L}^{\mu}(\mathbb{R})} \leq C|u|_{J_{L, 0}^{\mu}(\Omega)}
$$

Proof. Using Theorems 2.1 and 2.13, we have

$$
|\tilde{u}|_{J_{L}^{\mu}(\mathbb{R})}=|\tilde{u}|_{H^{\mu}(\mathbb{R})}=|u|_{H_{0}^{\mu}(\Omega)} \leq C|u|_{J_{L, 0}^{\mu}(\Omega)} .
$$


For the semi-norm on $H_{0}^{\mu}(\Omega)$ defined by (3), we have a fractional Poincaré-Friedrichs inequality for $H_{0}^{\mu}(\Omega)$.

Corollary 2.15 [Fractional Poincaré-Friedrichs] For $u \in H_{0}^{\mu}(\Omega)$, we have

$$
\|u\|_{L^{2}(\Omega)} \leq C|u|_{H_{0}^{\mu}(\Omega)}
$$

and for $0<s<\mu, s \neq n-1 / 2, n \in \mathbb{N}$

$$
|u|_{H_{0}^{s}(\Omega)} \leq C|u|_{H_{0}^{\mu}(\Omega)}
$$

Proof. The result follows from Theorem 2.10, Corollary 2.11, and the equivalence of norms, Theorem 2.13 .

In order to provide regularity estimates for functions $u \in H^{\mu}(\Omega)$ solving (1), we state an additional estimate for $\mu=n-1 / 2, n \in \mathbb{N}$.

Corollary 2.16 For $u \in H_{0}^{\mu}(\Omega), \mu=n-1 / 2, n \in \mathbb{N}$ and $0<\epsilon<1 / 2$, there exists a constant $C$ depending only upon $\epsilon, u$ such that

$$
\begin{aligned}
|u|_{H^{\mu-\epsilon}(\Omega)} & \leq C|u|_{J_{L, 0}^{\mu}(\Omega)}, \\
|u|_{H^{\mu-\epsilon}(\Omega)} & \leq C|u|_{J_{R, 0}^{\mu}(\Omega)} .
\end{aligned}
$$

Proof. As $\mu-\epsilon \neq n-1 / 2, n \in \mathbb{N}$, Theorem 2.13 implies

$$
|u|_{H^{\mu-\epsilon}(\Omega)} \leq C_{1}|u|_{J_{L, 0}^{\mu-\epsilon}(\Omega)}
$$

Using Corollary 2.11, we have

$$
|u|_{J_{L, 0}^{\mu-\epsilon}(\Omega)} \leq C_{2}|u|_{J_{L, 0}^{\mu}(\Omega)}
$$

Therefore, the stated result follows. The result for $J_{R, 0}^{\mu}(\Omega)$ follows analogously.

\section{Variational Formulation}

Let $\Omega=(0,1)$ and $0 \leq \beta<1$. Define $\alpha:=\frac{2-\beta}{2}$, so that $1 / 2<\alpha \leq 1$. In this section, we will show that there exists a unique variational solution of (1) in the space $H_{0}^{\alpha}(\Omega)$.

Problem 1 [Steady-State Fractional Advection Dispersion Equation] Given $\Omega=(0,1), f: \bar{\Omega} \rightarrow \mathbb{R}$, find $u: \bar{\Omega} \rightarrow \mathbb{R}$ such that

$$
\begin{aligned}
L u & =f, \quad \text { in } \Omega \\
u & =0, \quad \text { on } \partial \Omega
\end{aligned}
$$

where

$$
L u:=-D a\left(p_{0} D_{x}^{-\beta}+q_{x} D_{1}^{-\beta}\right) D u+b(x) D u+c(x) u
$$

$0 \leq \beta<1, a>0, b(x) \in C^{1}(\bar{\Omega}), c(x) \in C(\bar{\Omega})$ with $c-1 / 2 D b \geq 0$, and $p+q=1,0 \leq p, q \leq 1$. 
In order to derive a variational form of Problem 1, we assume that $u$ is a sufficiently smooth solution of (20)-(21), and multiply by an arbitrary $v \in C_{0}^{\infty}(\Omega)$ to obtain

$$
\int_{\Omega}-D a\left(p_{0} D_{x}^{-\beta}+q_{x} D_{1}^{-\beta}\right) D u v+b D u v+c u v d x=\int_{\Omega} f v d x .
$$

Integrating by parts in the first integral, and noting that $v=0$ on $\partial \Omega$ gives

$$
\int_{\Omega} a\left(p_{0} D_{x}^{-\beta}+q_{x} D_{1}^{-\beta}\right) D u D v+b D u v+c u v d x=\int_{\Omega} f v d x .
$$

Thus, we define the associated bilinear form $B: H_{0}^{\alpha}(\Omega) \times H_{0}^{\alpha}(\Omega) \rightarrow \mathbb{R}$ as

$$
B(u, v):=a p\left\langle{ }_{0} D_{x}^{-\beta} D u, D v\right\rangle+a q\left\langle{ }_{x} D_{1}^{-\beta} D u, D v\right\rangle+\langle b D u, v\rangle+(c u, v),
$$

where $(\cdot, \cdot)$ denotes the inner product on $L^{2}(\Omega)$, and $<\cdot, \cdot>$ the duality pairing of $H^{-\mu}(\Omega)$ and $H_{0}^{\mu}(\Omega)$, $\mu \geq 0$.

For a given $f \in H^{-\alpha}(\Omega)$, we define the associated linear functional $F: H_{0}^{\alpha}(\Omega) \rightarrow \mathbb{R}$ as

$$
F(v):=\langle f, v\rangle
$$

Note that the duality pairings in $(22)$ are well defined for $u, v \in H_{0}^{\alpha}(\Omega)$.

Thus, the Galerkin variational solution of (20)-(21) may be defined as follows.

Definition 3.1 [Variational Solution] A function $u \in H_{0}^{\alpha}(\Omega)$ is a variational solution of Problem 1 provided that

$$
B(u, v)=F(v), \quad \forall v \in H_{0}^{\alpha}(\Omega) .
$$

Using the results of Section 2, we show that there exists a unique solution to (24). To do this we begin by establishing coercivity and continuity of $B(\cdot, \cdot)$.

Lemma 3.1 The bilinear form $B(\cdot, \cdot)$ is coercive over $H_{0}^{\alpha}(\Omega)$, i.e. there exists a constant $C_{0}>0$ such that

$$
B(u, u) \geq C_{0}\|u\|_{H^{\alpha}(\Omega)}^{2}, \quad \forall u \in H_{0}^{\alpha}(\Omega)
$$

Proof. We have that

$$
\begin{aligned}
B(u, u) & =a p\left\langle{ }_{0} D_{x}^{-\beta} D u, D u\right\rangle+a q\left\langle{ }_{x} D_{1}^{-\beta} D u, D u\right\rangle+\langle(b D u+c u), u\rangle \\
& =a p\left\langle{ }_{0} D_{x}^{-\beta} D u, D u\right\rangle+a q\left\langle{ }_{x} D_{1}^{-\beta} D u, D u\right\rangle+\left\langle\left(c-\frac{1}{2} D b\right) u, u\right\rangle \\
& \geq a p\left\langle{ }_{0} D_{x}^{-\beta} D u, D u\right\rangle+a q\left\langle{ }_{x} D_{1}^{-\beta} D u, D u\right\rangle
\end{aligned}
$$

as $c-\frac{1}{2} D b \geq 0$.

Applying the semi-group and adjoint properties of the Riemann-Liouville fractional integral operators, we have

$$
B(u, u) \geq a\left\langle{ }_{0} D_{x}^{-\beta / 2} D u,{ }_{x} D_{1}^{-\beta / 2} D u\right\rangle .
$$

As $u=0$ on $\partial \Omega$,

$$
\begin{aligned}
{ }_{0} D_{x}^{-\beta / 2} D u & =\mathbf{D}^{\alpha} u, \\
{ }_{x} D_{1}^{-\beta / 2} D u & =-\mathbf{D}^{\alpha *} u .
\end{aligned}
$$


Thus

$$
B(u, u) \geq-a\left(\mathbf{D}^{\alpha} u, \mathbf{D}^{\alpha *} u\right)=a|u|_{J_{S, 0}^{\alpha}(\Omega)}^{2}
$$

Note that as $1 / 2<\alpha \leq 1$, Lemma 2.4 implies $\left(\mathbf{D}^{\alpha} u, \mathbf{D}^{\alpha *} u\right)<0$.

The semi-norm equivalence of $J_{S, 0}^{\alpha}(\Omega)$ and $H_{0}^{\alpha}(\Omega)$, Theorem 2.12, implies that

$$
B(u, u) \geq C|u|_{H^{\alpha}(\Omega)}^{2}
$$

By the fractional Poincaré-Friedrichs inequality, Corollary 2.15, we have

$$
|u|_{H^{\alpha}(\Omega)} \geq \tilde{C}\|u\|_{L^{2}(\Omega)}
$$

Therefore,

$$
B(u, u) \geq C\|u\|_{H^{\alpha}(\Omega)}^{2}
$$

To establish the continuity of $B(\cdot, \cdot)$, we make use of the following lemma.

Lemma 3.2 Let $b \in C^{1}(\bar{\Omega})$. Then for all $v \in H_{0}^{\alpha}(\Omega)$, there exists a constant $C$ depending only upon $b$ and $\alpha$ such that

$$
\|b v\|_{H^{\alpha}(\Omega)} \leq C\|v\|_{H^{\alpha}(\Omega)} .
$$

Proof. As $b \in C^{1}(\bar{\Omega})$, we can show that the linear mappings $T_{0}, T_{1}$ defined by

$$
T_{0}(v)=T_{1}(v)=b v
$$

are bounded. First, we have that $T_{0}: L^{2}(\Omega) \rightarrow L^{2}(\Omega)$ is bounded as

$$
\left\|T_{0}(v)\right\|_{L^{2}(\Omega)}^{2}=\|b v\|_{L^{2}(\Omega)}^{2} \leq\|b\|_{\infty}^{2}\|v\|_{L^{2}(\Omega)}^{2} .
$$

Next, we have that $T_{1}: H^{1}(\Omega) \rightarrow H^{1}(\Omega)$ is bounded as

$$
\begin{aligned}
\left\|T_{1}(v)\right\|_{H^{1}(\Omega)}^{2} & =\|b v\|_{H^{1}(\Omega)}^{2} \\
& =\|b v\|_{L^{2}(\Omega)}^{2}+\|b D v\|_{L^{2}(\Omega)}^{2}+\|v D b\|_{L^{2}(\Omega)}^{2} \\
& \leq\|b\|_{\infty}^{2}\|v\|_{H^{1}(\Omega)}^{2}+\|D b\|_{\infty}\|v\|_{L^{2}(\Omega)}^{2} \\
& \leq\left(\|b\|_{\infty}^{2}+\|D b\|_{\infty}^{2}\right)\|v\|_{H^{1}(\Omega)}^{2} .
\end{aligned}
$$

Therefore, using operator interpolation (see [11], p. 358),

$$
\begin{aligned}
\|b v\|_{H^{\alpha}(\Omega)} & \leq\left\|T_{1}\right\|^{\alpha}\left\|T_{0}\right\|^{1-\alpha}\|v\|_{H^{\alpha}(\Omega)} \\
& \leq C\|v\|_{H^{\alpha}(\Omega)},
\end{aligned}
$$

where $C$ depends only upon $b$ and $\alpha$.

Lemma 3.3 The bilinear form $B(\cdot, \cdot)$ is continuous on $H_{0}^{\alpha}(\Omega) \times H_{0}^{\alpha}(\Omega)$, i.e. there exists a constant $C_{1}$ such that

$$
|B(u, v)| \leq C_{1}\|u\|_{H^{\alpha}(\Omega)}\|v\|_{H^{\alpha}(\Omega)}, \quad \forall u, v \in H_{0}^{\alpha}(\Omega) .
$$

Proof. From the definition of $B$ we have

$$
|B(u, v)| \leq a p\left|\left(\mathbf{D}^{\alpha} u, \mathbf{D}^{\alpha *} v\right)\right|+a q\left|\left(\mathbf{D}^{\alpha *} u, \mathbf{D}^{\alpha} v\right)\right|+|\langle b D u, v\rangle|+|(c u, v)| .
$$


Using the equivalence of norms, Theorem 2.13, and Lemma 3.2,

$$
\begin{aligned}
|\langle b D u, v\rangle| & =\left|\left(\mathbf{D}^{\alpha} u, \mathbf{D}^{\beta / 2 *}(b v)\right)\right| \\
& \leq|u|_{J_{L}^{\alpha}(\Omega)}|b v|_{J_{R}^{\alpha}(\Omega)} \\
& \leq C\|u\|_{H^{\alpha}(\Omega)}\|b v\|_{H^{\alpha}(\Omega)}, \\
& \leq C\|u\|_{H^{\alpha}(\Omega)}\|v\|_{H^{\alpha}(\Omega)} .
\end{aligned}
$$

Thus,

$$
\begin{aligned}
|B(u, v)| \leq & a p\|u\|_{J_{L}^{\alpha}(\Omega)}\|v\|_{J_{R}^{\alpha}(\Omega)}+a q\|u\|_{J_{R}^{\alpha}(\Omega)}\|v\|_{J_{L}^{\alpha}(\Omega)} \\
& +C\|u\|_{H^{\alpha}(\Omega)}\|v\|_{H^{\alpha}(\Omega)}+\|c\|_{\infty}\|u\|_{L_{2}(\Omega)}\|v\|_{L_{2}(\Omega)} \\
\leq & C\|u\|_{H^{\alpha}(\Omega)}\|v\|_{H^{\alpha}(\Omega)} .
\end{aligned}
$$

Lemma 3.4 The linear functional $F(\cdot)$ is continuous over $H_{0}^{\alpha}(\Omega)$.

Proof. The result follows from the fact that

$$
F(v)=\langle f, v\rangle \leq\|f\|_{H^{-\alpha}(\Omega)}\|v\|_{H^{\alpha}(\Omega)} .
$$

Theorem 3.5 There exists a unique solution $u \in H_{0}^{\alpha}(\Omega)$ to $(24)$ satisfying

$$
\|u\|_{H^{\alpha}(\Omega)} \leq C\|f\|_{H^{-\alpha}(\Omega)} .
$$

Proof. By Lemmas 3.1, 3.3, and 3.4, B, F satisfy the hypotheses of the Lax-Milgram theorem, from which existence and uniqueness of a solution to (24) immediately follow. The estimate (30) is obtained from (25), (26), and (29).

\section{Finite Element Convergence Estimates}

Let $S_{h}$ denote a partition of $\Omega$ such that $\bar{\Omega}=\left\{\cup K: K \in S_{h}\right\}$. Assume that there exist positive constants $c_{1}, c_{2}$ such that $c_{1} h \leq h_{K} \leq c_{2} h$, where $h_{K}$ is the width of the subinterval $K$, and $h=\max _{K \in S_{h}} h_{K}$.

Let $P_{k}(K)$ denote the space of polynomials of degree less than or equal to $k$ on $K \in S_{h}$. Associated with $S_{h}$, define the finite-dimensional subspace $X_{h} \subset H_{0}^{\alpha}(\Omega)$ as

$$
X_{h}:=\left\{v \in H_{0}^{\alpha}(\Omega) \cap C^{0}(\bar{\Omega}):\left.v\right|_{K} \in P_{m-1}(K), \forall K \in S_{h}\right\} .
$$

Denote by $\mathcal{I}^{h} u$ the piecewise polynomial interpolant of $u$ in $S_{h}$.

Let $u_{h}$ be the solution to the finite-dimensional variational problem

$$
B\left(u_{h}, v_{h}\right)=F\left(v_{h}\right), \quad \forall v_{h} \in X_{h} .
$$


We define the energy norm associated with (24) as

$$
\|u\|_{E}:=B(u, u)^{1 / 2}
$$

Note that from (25) and (26) we have norm equivalence of $\|\cdot\|_{H^{\alpha}(\Omega)}$ and $\|\cdot\|_{E}$.

Theorem 4.1 Let $u$ denote the solution to (24). There exists a unique solution to (31) which satisfies the estimate

$$
\begin{aligned}
\left\|u-u_{h}\right\|_{E} & \leq C_{I} \inf _{v \in X_{h}}\|u-v\|_{E} \\
& \leq C_{I}\left\|u-\mathcal{I}^{h} u\right\|_{E} .
\end{aligned}
$$

Proof. Existence and uniqueness follow from the fact that $X_{h}$ is a subset of the space $H_{0}^{\alpha}(\Omega)$, and thus (31) satisfies the hypotheses of the Lax-Milgram lemma over the finite-dimensional subspace $X_{h}$. The estimate (33) is a result of Ceá's lemma.

The finite-dimensional subspace $X_{h}$ and the interpolant $\mathcal{I}^{h} u$ are chosen specifically so that they satisfy an approximation property over subspaces of $H^{m}(\Omega)$. That is to say that $\mathcal{I}^{h} u$ satisfies the following theorem [3].

Theorem 4.2 [Approximation Property] Let $u \in H^{r}(\Omega), 0<r \leq m$, and $0 \leq s \leq r$. Then there exists a constant $C_{A}$ depending only on $\Omega$ such that

$$
\left\|u-\mathcal{I}^{h} u\right\|_{H^{s}(\Omega)} \leq C_{A} h^{r-s}\|u\|_{H^{r}(\Omega)}
$$

We can combine the previous results into an estimate for $e:=u-u_{h}$ in the energy norm.

Corollary 4.3 Let $u \in H_{0}^{\alpha}(\Omega) \cap H^{r}(\Omega)(\alpha \leq r \leq m)$ solve (24), and $u_{h}$ solve (31). Then there exists a constant $C$ such that the error $e=u-u_{h}$ satisfies

$$
\|e\|_{H^{\alpha}(\Omega)} \leq C h^{r-\alpha}\|u\|_{H^{r}(\Omega)} .
$$

Proof. From Theorem 4.1, we have that the error satisfies

$$
\|e\|_{E} \leq C_{I}\left\|u-\mathcal{I}^{h} u\right\|_{E}
$$

Applying the approximation property and continuity yields

$$
\|e\|_{E} \leq \sqrt{C_{1}} C_{I} C_{A} h^{r-\alpha}\|u\|_{H^{r}(\Omega)}
$$

Finally, we obtain (35) via the norm equivalence of $\|\cdot\|_{H^{\alpha}(\Omega)}$ and $\|\cdot\|_{E}$.

We now apply the Aubin-Nitsche trick to obtain a convergence estimate in the $L^{2}$ norm. First, we must make an assumption concerning the regularity of the solution to the adjoint problem

$$
\begin{aligned}
-D\left(q_{0} D_{x}^{-\beta}+p_{x} D_{1}^{-\beta}\right) a D w-D(b(x) w)+c(x) w & =g \quad \text { in } \Omega \\
w & =0, \quad \text { on } \partial \Omega .
\end{aligned}
$$


Assumption 4.1 For $w$ solving (37) with $g \in L^{2}(\Omega)$, we have

$$
\|w\|_{H^{2 \alpha}(\Omega)} \leq C_{E}\|g\|_{L^{2}(\Omega)} .
$$

Theorem 4.4 Let $u \in H_{0}^{\alpha}(\Omega) \cap H^{r}(\Omega)(\alpha \leq r \leq m)$ solve (24), and $u_{h}$ solve (31). Then, under Assumption 4.1, there exists a constant $C$ such that the error $e=u-u_{h}$ satisfies

$$
\begin{aligned}
\|e\|_{L^{2}(\Omega)} & \leq C h^{r}\|u\|_{H^{r}(\Omega)}, \quad \alpha \neq 3 / 4, \\
\|e\|_{L^{2}(\Omega)} & \leq C h^{r-\epsilon}\|u\|_{H^{r}(\Omega)}, \quad \alpha=3 / 4, \quad 0<\epsilon<1 / 2 .
\end{aligned}
$$

Proof. Introduce $w$ as the solution to (37) with $g=e=u-u_{h} \in L^{2}(\Omega)$. Then $w$ satisfies the variational form

$$
B(v, w)=(e, v), \quad \forall v \in H_{0}^{\alpha}(\Omega)
$$

and the regularity estimate

$$
\|w\|_{H^{2 \alpha}(\Omega)} \leq C_{E}\|e\|_{L^{2}(\Omega)} .
$$

Substituting $v=e$ in (39), and applying Galerkin orthogonality, we have

$$
\begin{aligned}
\|e\|_{L^{2}(\Omega)}^{2} & =B(e, w) \\
& =B\left(e, w-\mathcal{I}^{h} w\right) \\
& \leq C_{1}\|e\|_{H^{\alpha}(\Omega)}\left\|w-\mathcal{I}^{h} w\right\|_{H^{\alpha}(\Omega)} \\
& \leq C_{1} C_{A} h^{\alpha}\|e\|_{H^{\alpha}(\Omega)}\|w\|_{H^{2 \alpha}(\Omega)} \\
& \leq C_{1} C_{A} C_{E} h^{\alpha}\|e\|_{H^{\alpha}(\Omega)}\|e\|_{L^{2}(\Omega)} .
\end{aligned}
$$

Therefore, dividing through by $\|e\|_{L^{2}(\Omega)}$ yields the estimate

$$
\|e\|_{L^{2}(\Omega)} \leq C_{1} C_{A} C_{E} h^{\alpha}\|e\|_{E}
$$

and applying (35) we obtain (38). The stated result for $\alpha=3 / 4$ follows analogously.

Note that for $\alpha=3 / 4$, the $L^{2}$ convergence rate is effectively $r$ for $u \in H^{r}(\Omega)$.

\section{Numerical Calculations for Piecewise Linear Polynomi-}

\section{als}

Let $\Omega=(0,1)$ and let $X_{h}$ denote the space of continuous piecewise linear polynomials over $S_{h}$, i.e. $m=2$. In this section, we present numerical calculations which support the error estimates in the Corollary 4.3 and Theorem 4.4 .

Example 1. Let $u=x^{2}, p=1, q=0, \beta=1 / 2, \alpha=3 / 4, a=1$, and $b=c=0$. Then, $u$ is the exact solution to the boundary value problem

$$
\begin{aligned}
-D_{0} D_{x}^{-1 / 2} D u & =\frac{-2 \sqrt{x}}{\Gamma(3 / 2)} \\
u(0) & =0 \\
u(1) & =1 .
\end{aligned}
$$


As $u \in H^{2}(\Omega)$, the results of Corollary 4.3 and Theorem 4.4 predict a convergence rate of 2 in the $L^{2}$ norm and 5/4 in the energy norm. Table 1 includes numerical calculations over a regular partition of $[0,1]$ which support the predicted rates of convergence.

\begin{tabular}{|c||c|c||c|c|}
\hline$h$ & $\left\|u-u_{h}\right\|_{L^{2}(\Omega)}$ & $\begin{array}{c}\text { cvge. } \\
\text { rate }\end{array}$ & $\left\|u-u_{h}\right\|_{J_{L}^{3 / 4}(\Omega)}$ & $\begin{array}{c}\text { cvge. } \\
\text { rate }\end{array}$ \\
\hline $1 / 4$ & $9.319448 \cdot 10^{-3}$ & & $6.078802 \cdot 10^{-2}$ & \\
\hline $1 / 8$ & $2.287113 \cdot 10^{-3}$ & 2.03 & $2.506011 \cdot 10^{-2}$ & 1.28 \\
\hline $1 / 16$ & $5.626150 \cdot 10^{-4}$ & 2.02 & $1.049400 \cdot 10^{-2}$ & 1.27 \\
\hline $1 / 32$ & $1.391495 \cdot 10^{-4}$ & 2.02 & $4.354633 \cdot 10^{-3}$ & 1.26 \\
\hline $1 / 64$ & $3.439341 \cdot 10^{-5}$ & 2.02 & $1.825171 \cdot 10^{-3}$ & 1.25 \\
\hline
\end{tabular}

Table 1: Experimental error results for Example 1.

Example 2. Let $u=x^{2}-x^{3}, \beta=1 / 2, \alpha=3 / 4, a=2$, and $b=c=1$. Then, $u$ is the exact solution to the boundary value problem

$$
\begin{aligned}
-2 D\left(p_{0} D_{x}^{-1 / 2}+q_{x} D_{1}^{-1 / 2}\right) D u+D u+u & =f \\
u(0) & =0 \\
u(1) & =0 .
\end{aligned}
$$

As $u \in H^{2}(\Omega)$, Theorem 4.4 predicts a rate of convergence of 2 in the $L^{2}$ norm. Table 2 includes numerical calculations over a regular partition of $[0,1]$ which support the predicted rates of convergence for different values of $p$ and $q$.

\begin{tabular}{|c||c|c||c|c||c|c|}
\hline$h$ & $\left\|u-u_{h}\right\|_{L^{2}(\Omega)}$ & cvge. & $\left\|u-u_{h}\right\|_{L^{2}(\Omega)}$ & cvge. & $\left\|u-u_{h}\right\|_{L^{2}(\Omega)}$ & cvge. \\
& $p=1, q=0$ & rate & $p=0, q=1$ & rate & $p=0.5, q=0.5$ & rate \\
\hline $1 / 4$ & $1.291090 \cdot 10^{-2}$ & & $1.036369 \cdot 10^{-2}$ & & $8.307486 \cdot 10^{-3}$ & \\
\hline $1 / 8$ & $3.230796 \cdot 10^{-3}$ & 2.00 & $2.338583 \cdot 10^{-3}$ & 2.15 & $1.868104 \cdot 10^{-3}$ & 2.15 \\
\hline $1 / 16$ & $8.123848 \cdot 10^{-4}$ & 1.99 & $5.623415 \cdot 10^{-4}$ & 2.06 & $4.274829 \cdot 10^{-4}$ & 2.12 \\
\hline $1 / 32$ & $2.049679 \cdot 10^{-4}$ & 1.99 & $1.387338 \cdot 10^{-4}$ & 2.02 & $1.003665 \cdot 10^{-4}$ & 2.09 \\
\hline $1 / 64$ & $5.189776 \cdot 10^{-5}$ & 1.98 & $3.476001 \cdot 10^{-5}$ & 2.00 & $2.434186 \cdot 10^{-5}$ & 2.04 \\
\hline
\end{tabular}

Table 2: Experimental error results for Example 2.

Example 3. Let $u=x^{\lambda}-x, p=1, q=0, a=1, b=c=0$ and $0 \leq \beta<1$. Then, $u$ is the exact solution to the boundary value problem

$$
-D_{0} D_{x}^{-\beta} D u=\frac{-\Gamma(\lambda+1) x^{\lambda+\beta-2}}{\Gamma(\lambda+\beta-1)}+\frac{x^{\beta-1}}{\Gamma(\beta)}
$$




$$
\begin{aligned}
& u(0)=0 \\
& u(1)=0 .
\end{aligned}
$$

Suppose that $\lambda<3 / 2$. Then $u \in H^{r-\epsilon}(\Omega)$ where $r=\lambda+1 / 2$, and so the result of Theorem 4.4 predicts a rate of convergence of $r$ in the $L^{2}$ norm. For this example, we provide two sets of calculations,

$$
\begin{aligned}
& \text { (A) } \lambda=1.1, \beta=0.8, \alpha=0.6, r=1.6 \\
& \text { (B) } \lambda=1.4, \beta=0.4, \alpha=0.8, r=1.9
\end{aligned}
$$

Table 3 contains numerical results which support the predicted rate convergence.

\begin{tabular}{|c||c|c||c|c|}
\hline$h$ & $\left\|u-u_{h}\right\|_{L^{2}(\Omega)}$ & cvge. & $\left\|u-u_{h}\right\|_{L^{2}(\Omega)}$ & cvge. \\
$(\mathrm{A})$ & rate & $\mathrm{B})$ & rate \\
\hline $1 / 4$ & $3.815961 \cdot 10^{-3}$ & & $5.508758 \cdot 10^{-3}$ & \\
\hline $1 / 8$ & $1.064472 \cdot 10^{-3}$ & 1.84 & $1.496356 \cdot 10^{-3}$ & 1.88 \\
\hline $1 / 16$ & $3.458776 \cdot 10^{-4}$ & 1.62 & $4.052072 \cdot 10^{-4}$ & 1.88 \\
\hline $1 / 32$ & $1.143203 \cdot 10^{-4}$ & 1.60 & $1.098377 \cdot 10^{-4}$ & 1.88 \\
\hline $1 / 64$ & $3.772030 \cdot 10^{-5}$ & 1.60 & $2.986717 \cdot 10^{-5}$ & 1.88 \\
\hline
\end{tabular}

Table 3: Experimental error results for Example 3.

\section{A Riemann-Liouville Fractional Integral Operators}

We define the fractional integral operators in terms of the Riemann-Liouville definition given in $[9,10]$.

Definition A.1 [Left Riemann-Liouville Fractional Integral] Let $u$ be a function defined on $(a, b)$, and $\sigma>0$. Then the left Riemann-Liouville fractional integral of order $\sigma$ is defined to be

$$
{ }_{a} D_{x}^{-\sigma} u(x):=\frac{1}{\Gamma(\sigma)} \int_{a}^{x}(x-s)^{\sigma-1} u(s) d s .
$$

Definition A.2 [Right Riemann-Liouville Fractional Integral] Let $u$ be a function defined on $(a, b)$, and $\sigma>0$. Then the right Riemann-Liouville fractional integral of order $\sigma$ is defined to be

$$
{ }_{x} D_{b}^{-\sigma} u(x):=\frac{1}{\Gamma(\sigma)} \int_{x}^{b}(s-x)^{\sigma-1} u(s) d s .
$$

With these definitions, we note some of the properties of the Riemann-Liouville fractional integral operators, as outlined in [9, 10].

Property A.1 [Semigroup Property] The left and right Riemann-Liouville fractional integral operators follow the properties of a semigroup, i.e. for $u \in L^{p}[a, b]$ for $p \geq 1$,

$$
\begin{aligned}
& { }_{a} D_{x}^{-\mu}{ }_{a} D_{x}^{-\sigma} u(x)={ }_{a} D_{x}^{-\mu-\sigma} u(x), \forall x \in[a, b], \forall \mu, \sigma>0, \\
& { }_{x} D_{b}^{-\mu}{ }_{x} D_{b}^{-\sigma} u(x)={ }_{x} D_{b}^{-\mu-\sigma} u(x), \forall x \in[a, b], \forall \mu, \sigma>0 .
\end{aligned}
$$


Property A.2 [Adjoint Property] The left and right Riemann-Liouville fractional integral operators are adjoints in the $L^{2}$ sense, i.e. for all $\sigma>0$,

$$
\left({ }_{a} D_{x}^{-\sigma} u, v\right)_{L^{2}(a, b)}=\left(u,{ }_{x} D_{b}^{-\sigma} v\right)_{L^{2}(a, b)}, \forall u, v \in L^{2}(a, b) .
$$

Property A.3 [Fourier Transform Property] Let $\sigma>0, u \in L^{p}(\mathbb{R}), \mathrm{p} \geq 1$. The Fourier transform of the left and right Riemann-Liouville fractional integrals satisfy the following,

$$
\begin{aligned}
\mathcal{F}\left({ }_{-\infty} D_{x}^{-\sigma} u(x)\right) & =(i \omega)^{-\sigma} \hat{u}(\omega), \\
\mathcal{F}\left({ }_{x} D_{\infty}^{-\sigma} u(x)\right) & =(-i \omega)^{-\sigma} \hat{u}(\omega),
\end{aligned}
$$

where $\hat{u}(\omega)$ denotes the Fourier transform of $u$,

$$
\hat{u}(\omega)=\int_{\mathbb{R}} e^{-i \omega x} u(x) d x .
$$

Definition A.3 [Left Riemann-Liouville Fractional Derivative] Let $u$ be a function defined on $\mathbb{R}, \mu>0, n$ be the smallest integer greater than $\mu(n-1 \leq \mu<n)$, and $\sigma=n-\mu$. Then the left fractional derivative of order $\mu$ is defined to be

$$
\mathbf{D}^{\mu} u:={ }_{-\infty} D_{x}^{\mu} u=D^{n}{ }_{-\infty} D_{x}^{-\sigma} u(x)=\frac{1}{\Gamma(\sigma)} \frac{d^{n}}{d x^{n}} \int_{-\infty}^{x}(x-\xi)^{\sigma-1} u(\xi) d \xi .
$$

Definition A.4 [Right Riemann-Liouville Fractional Derivative] Let $u$ be a function defined on $\mathbb{R}, \mu>0, n$ be the smallest integer greater than $\mu(n-1 \leq \mu<n)$, and $\sigma=n-\mu$. Then the right fractional derivative of order $\mu$ is defined to be

$$
\mathbf{D}^{\mu *} u:={ }_{x} D_{\infty}^{\mu} u=(-D)^{n}{ }_{x} D_{\infty}^{-\sigma} u(x)=\frac{(-1)^{n}}{\Gamma(\sigma)} \frac{d^{n}}{d x^{n}} \int_{x}^{\infty}(\xi-x)^{\sigma-1} u(\xi) d \xi .
$$

Note. If $\overline{\operatorname{supp}(u)} \subset(a, b)$, then $\mathbf{D}^{\mu} u={ }_{a} D_{x}^{\mu} u$ and $\mathbf{D}^{\mu *} u={ }_{x} D_{b}^{\mu} u$, where ${ }_{a} D_{x}^{\mu}$ and ${ }_{x} D_{b}^{\mu} u$ are the left and right Riemann-Liouville fractional derivatives of order $\mu$ [9].

Property A.4 The left (right) Riemann-Liouville fractional derivative of order $\mu$ acts as a left inverse of the left (right) Riemann-Liouville fractional integral of order $\mu$, i.e.

$$
\begin{aligned}
{ }_{a} D_{x}^{\mu}{ }_{a} D_{x}^{-\mu} u(x) & =u(x), \\
{ }_{x} D_{b}^{\mu}{ }_{x} D_{b}^{-\mu} u(x) & =u(x), \forall \mu>0 .
\end{aligned}
$$

Property A.5 [Fourier Transform Property] Let $\mu>0, u \in C_{0}^{\infty}(\Omega), \Omega \subset \mathbb{R}$. The Fourier transform of the left and right Riemann-Liouville fractional derivatives satisfy the following,

$$
\begin{aligned}
\mathcal{F}\left({ }_{-\infty} D_{x}^{\mu} u(x)\right) & =(i \omega)^{\mu} \hat{u}(\omega), \\
\mathcal{F}\left({ }_{x} D_{\infty}^{\mu} u(x)\right) & =(-i \omega)^{\mu} \hat{u}(\omega) .
\end{aligned}
$$

Property A.6 Let $\mu>0, u \in C^{\infty}(\Omega), \Omega \subset \mathbb{R}$. The following composition rule holds for the left (right) Riemann-Liouville fractional integral and fractional derivative operators.

$$
\begin{aligned}
& { }_{a} D_{x}^{-\mu}{ }_{a} D_{x}^{\mu} u(x)=u(x), \quad \forall u(x) \text { such that } \overline{\operatorname{supp}(u)} \subset(a, \infty), \\
& { }_{x} D_{b}^{-\mu}{ }_{x} D_{b}^{\mu} u(x)=u(x), \quad \forall u(x) \text { such that } \overline{\operatorname{supp}(u)} \subset(-\infty, b) .
\end{aligned}
$$




\section{References}

[1] R.A. Adams. Sobolev Spaces. Academic Press, New York, 1975.

[2] D.A. Benson, S.W. Wheatcraft, and M.M. Meerschaeert. The fractional order governing equations of levy motion. Water Resour. Res., 36:1413-1423, 2000.

[3] S. Brenner and L.R. Scott. The Mathematical Theory of Finite Element Methods. Springer-Verlag, New York, 1994.

[4] B.A. Carreras, V.E. Lynch, and G.M. Zaslavsky. Anomalous diffusion and exit time distribution of particle tracers in plasma turbulence models. Phys. Plasmas, 8(12):5096-5103, 2001.

[5] G.J. Fix and J.P. Roop. Least squares finite element solution of a fractional order two-point boundary value problem. Computers Math. Applic., to appear, 2004.

[6] J.W. Kirchner, X. Feng, and C. Neal. Fractal streamchemistry and its implications for contaminant transport in catchments. Nature, 403:524-526, 2000.

[7] F. Mainardi. Fractional calculus. In A. Carpinteri and F. Mainardi, editors, Fractals and Fractional Calculus in Continuum Mechanics, New York, 1997. Springer-Verlag.

[8] K.B. Oldham and J. Spanier. The Fractional Calculus. Academic Press, New York, 1974.

[9] I. Podlubny. Fractional Differential Equations. Academic Press, New York, 1999.

[10] S.G. Samko, A.A. Kilbas, and O.I. Marichev. Fractional Integrals and Derivatives: Theory and Applications. Gordon and Breach, New York, 1993.

[11] Ch. Schwab. p- and hp- Finite Element Methods: Theory and Applications in Solid and Fluid Mechanics. Oxford University Press, New York, 1998.

[12] M.F. Shlesinger, B.J. West, and J. Klafter. Lévy dynamics of enhanced diffusion: Application to turbulence. Phys. Rev. Lett., 58(11):1100-1103, 1987.

[13] I.M. Sokolov, J. Klafter, and A. Blumen. Fractional kinetics. Physics Today, Nov.:48-53, 2002.

[14] G.M. Zaslavsky, D.Stevens, and H. Weitzner. Self-similar transport in incomplete chaos. Phys. Rev. E, 48(3):1683-1694, 1993. 